\title{
Influence Technology of Wireless Sensors Network communication
}

\author{
Alaulddin Adel Albla \\ Phd, Alhikma college University \\ DOI: 10.29322/IJSRP.11.08.2021.p11659 \\ http://dx.doi.org/10.29322/IJSRP.11.08.2021.p11659
}

\section{INTRODUCTION}

$\mathrm{W}$ ireless Sensor Network, WSN) are distributed systems consisting of sensors connected by a wireless communication network. The application of wireless sensor networks is based on the use of small, cheap and multifunctional platforms that independently form an ad hoc wireless network and deliver the data collected from the sensor to the user. Wireless sensor networks are used in ecology, e-health, smart traffic and other areas.

\section{WHAT IS WSN}

WSN consist of spatially distributed and autonomous sensors to monitor the physical or environmental conditions in which they are located. Their main purpose is to collect, process and deliver measured information from the environment to end users. Appropriate types of passive or active sensors that are part of multifunctional platforms, ie. Sensor Nodes (SN). Sensor nodes enable storage and processing of collected data and communication with the IoT platform. ${ }^{1}$

In practice, they are most often implemented as data-centric systems, and rarely as address-centric systems.

The wireless sensor network consists of battery-powered modules that are essentially Snods. ${ }^{2}$ The architecture of these modules consists of:

- sensor: data generator

- radio transceiver: transmits its own or forwards through the network data received from its neighbors (routes data)

- one or more processors: controls the operation of sensors and transceivers, processes data, and implement network and routing protocols.

In most cases, sensor networks are implemented as datacentric, and not as address-centric systems. This means that queries are directed to a region made up of a topologically arranged group (cluster) of sensors, and not to a specific sensor address. Within a single cluster, there is a single aggregator node,

\footnotetext{
${ }^{1}$ Bežične senzorske mreže u poljoprivredi (Wireless sensor networks in agriculture), A. Višnjički, Beograd,2020. https://raf.edu.rs/docs/Diplomski_radovi/Aleksandar_Visnjicki_ Diplomski_rad.pdf,
}

which collects data from Snods associated with that cluster, analyzes it, aggregates it, and, after the roll call, passing over.

Essencially, the summary analysis of local data is performed by the node aggregator within the cluster. This significantly reduces the requirements related to communication bandwidth. Data aggregation increases the level of sensor measurement accuracy, and at the same time incorporates data redundancy, which compensates for failures in nodes. Analyzing the work of the sensor network, we notice that a number of information process techniques are used for:

- manipulation and analysis of sensory data

- extraction of significant features of the monitored environment

- efficient storage and transmission of important information.

\section{HARDWARE}

The hardware of the WSN includes microprocessors, memory, sensors, D / A converters, data transmitters, controllers that connect all the individual parts into a whole and power sources. With the increasing efficiency of chips and their miniaturization, there is a trend, that the efficiency of microcontrollers increases and the functionality is maintained at a similar level. The average microcontroller in such systems consumes about $1 \mathrm{~mW}$ of energy while operating at about $10 \mathrm{MHz}$. Most circuits are inactive most of the time, so standby power consumption drops to approximately $1 \mu \mathrm{W}$. If an individual in the WSN system is active for about $1 \%$ of the time, then the average consumption drops to approximately a few $\mu \mathrm{W}$.

Energy for work can be obtained in many different ways. Solar energy is used, for which the following calculation applies: the solar cell generates approximately $10 \mathrm{~mW}$ of energy per square centimeter in the open space, and approximately $10-100 \mu \mathrm{W}$ indoors. Mechanical energy sources can also be used, such as vibrations, if the sensors are located in a convenient location. This way of obtaining energy generates about $100 \mu \mathrm{W}$ of energy. Memory in such systems is limited. Typically for them is $1 \mathrm{Kbytes}$ of RAM, and less than 100Kbytes of ROM. This memory takes up most of the chip, and also consumes most of the energy reserves. Because of this, manufacturers often offer memory on a separate chip. ${ }^{3}$

${ }^{2}$ http://es.elfak.ni.ac.rs/rmif/Materijal/Novo\%20Materijal/Pogl22\%20Wireless\%20Sensor\%20Networks-ekstenzija8.doc

${ }^{3}$ Bežične senzorske mreže (Wireless sensor networks), Ivor Grubišić,

https://www.google.rs/url?sa=t\&rct=j\&q=\&esrc=s\&source=web 
Microsensors are the "eyes" and "ears" of the WSN system. WSN systems must be susceptible to amortization of changes in environmental conditions, since many materials change their electrical characteristics with changing environmental conditions. The first applications were military, but today a large number of non-military applications are being developed, ranging from infrastructural security to industrial sensores.

Sensors are built to predict changes in these conditions over a range. Increasingly, the so-called "Micromechanical systems" (MEMS), which can be cut into silicon, such as transistors, and are used as sensors for a wide variety of forces, chemical reactions and changes in the environment. At the same time, they are hundreds of times cheaper than expensive piezo-electric sensors, and they ensure high precision. They were first used commercially in the auto industry, as a sensor for opening airbags.

\section{Applications \\ Infrastructure security}

Facilities of crucial importance must be under appropriate surveillance in order to be able to react in a timely manner in the event of an enemy attack. Networks of video, acoustic and other sensors must be developed around these facilities. Improved reporting, detection, reduction of the number of false alarms can be achieved by communicating with a larger number of sensors. Although fixed networks connected to fixed communications can well protect the facilities in question, Ad hoc networks are even more flexible and more effective at reporting when needed. Sensor networks can also be used to detect chemical, nuclear and biological attack.

\section{Environmental and habitat monitoring}

The Center for Embedded Network Sensing (CENS), Los Angeles, and CA are companies that have the upper hand in developing sensor networks that serve to monitor habitat and the environment. Environmental sensors are used to study the response of vegetation to climate change and disease, while acoustic and image processing sensors can identify, monitor, and measure populations of birds and other species. A large sensor network called the System for the Vigilance of the Amazon (SIVAM) monitors large areas of the Amazon rainforest for the purpose of combating drug transportation and air traffic control. It consists of a large number of different sensors that are connected. These sensors are radars, image processing sensors and environmental sensors. Image sensors are located in space, radars are located on aircraft and environmental sensors are located on the ground. The communication network operates at different speeds. Maximum speeds are present in the transmission of sensor data located in aircraft and satellites while the slow network is applied in the transmission of sensor information located on the ground.

\section{Sensors in industry}

The industry has long been interested in the application of a wide variety of sensors, as this contributes to reducing costs and improving maintenance, as well as improving the performance of machines. Monitoring the behavior of machines, in a way that assumes vibrations and the state of lubricants and the insertion of sensors in areas inaccessible to humans are examples of the application of sensor applications in industry.

Remote wireless sensors that enable production management are particularly effective, provided that they ensure and maintain subordination to the general safety of production while reducing production costs. One example of a sensor in the industry is spectral sensors, which are a good replacement for existing instrumentation. They can be simple optical sensors such as optocouplers and $\mathrm{pH}$ meters, all the way to real spectral devices that can function as spectrometers, optical sensors can replace instruments and measure material characteristics and perform compositional measurements. Optical feel is made possible by miniaturization as a cheap electric pair or field. The essence of such sensors is that they enable multiple or matrix measurements: the measurements of hundreds or thousands of sensors are recorded in a database that can then be re-examined, and in this way real-time information is obtained.

\section{Traffic control}

Sensor networks have been used in traffic for a long time to detect vehicles and traffic lights. Such sensors are usually buried or raised. Surveillance cameras are also used in some places, most often transport vehicles. The information is sent to the operator at central locations. Such sensors and the connections between them are expensive, and such devices are placed only at certain points. Cheap wireless Ad-hoc networks are supposed to change the global picture of traffic in the world. Such sensors with built-in communication can be deployed at all traffic intersections to measure speed and count vehicles. The sensors will communicate with neighboring sensors and will provide a global traffic image that can be questioned by an operator (human or computer) who then generates a control signal. The second, more radical approach is about how each vehicle will have sensors on it, and the sensors on passing cars will communicate with each other about the traffic situation, in order to avoid traffic jams, etc.

\section{Signal cooperation and information processing}

Nodes in sensor networks work together to generate useful information. Signal cooperation and information processing over the network is a new area in research and relates to the connection of distributed information. Processing information from multiple sensors regularly provides more complete information but requires more sources of information and thus higher energy requirements. Less communication information is lost at lower levels, but a larger frequency range is required. ${ }^{4}$

When a node receives information from another node, then that information must be combined and linked to local information. Linking rules range from simple, like choosing the best result, to complex model-based techniques that consider how information is generated. There is also a compromise of performance and robustness. Simple connection rules contribute

4 Al-Karaki, J.N., Kamal, A.E. (2004) Routing techniques in wireless sensor networks: A survey. IEEE Wireless Communications, 11(6): 6-28
$\& c d=\& v e d=2$ ahUKEwiLjuyzjeDxAhXPh_OHHeUPCzI4ChAWe gQICRAA\&url=http $\% 3 \mathrm{~A} \% 2 \mathrm{~F} \% 2 \mathrm{Fwww}$. fer.unizg.hr\%2F_downl oad $\% 2$ Frepository $\% 2$ FWireless $\% 2520$ Sensor $\% 2520$ Networks.d oc\&usg=AOvVaw3tN9-PAcib4hdbYAQ01FLX 
to robustness, while more complex rules may depend on lower levels of the system. In a network environment, information can arrive after multiple paths. The linking algorithm must then be able to know whether the information in question needs to be considered, in order to avoid repeatability. Keeping track of information is a good solution in high processing power networks but in ad hoc networks this is not applicable.

Sensor networks are often used for tracking, detection and classification. Data aggregation is a significant problem when we have multiple goals in a small area. Each node must combine its environmental measurements with an individual goal. Finally, an information detected by one node must be combined with information detected by other nodes to avoid repeatability and allow connectivity. Performing optimal aggregation is computationally very expensive and requires a significant frequency range. It follows from the above that a compromise between performance and the sources used is also required to aggregate the data, and distributed data algorithms are intended for sensor networks. ${ }^{5}$ The next problem is how to reach the conditions of self-failure and stability of the network with its maximum service life. Dense networks of low-cost sensors allow spatial sampling without the need for expensive algorithms. These algorithms must be asynchronous, so that processing speed and communication can be changed and even disappear, and reestablished.

\section{INTERNET CONNECTION}

The sensor part of our system communicates via the Internet with the server to which all measurements measured by the sensors are sent. Their communication is based on the server sending queries to the microcontroller, which individually calls one sensor at a time, downloads its measurement data and sends it to the server. In order to enable that, it is necessary to provide the microcontroller with internet access via a GSM module.

\section{TCP / IP (Transmission Control Protocol/Internet Protocol) communication of servers and systems}

The software communicates with the system, retrieves the collected data from the sensors, archives them and prepares reports. The sensor in the field must have a predefined server ip address where it will send data packages and report its ip address within a certain period of time. When the device contacts the server from its new ip address, the server confirms that it has accepted the communication request. After that, the device confirms that it has started a session with the server and their communication can begin. ${ }^{6}$ The sensor reports to the server with a message containing:
- Ordinal number of measurements

- Time when the sensor sent measurement results

- Measurement results and add to the database

If the provider changes the ip address of the device in the field, sensor responds from its new ip address according to a predefined procedure and continues communication with the server.

The optimal layout of mobile sensor nodes is important mainly because of the energy consumption of these nodes because, as can be seen in all three examples, a large number of sensor nodes are required for one route to be fully "covered". Also, the conclusion immediately arises that frequently changing sensor nodes would not be profitable precisely because of the large number of them. That is why in wireless sensor networks, the emphasis is always placed on sensor nodes and their consumption is analyzed. The analysis of consumption determines which of the sensor nodes has the highest consumption and on the basis of that node the conditions are set and the duration of the sensor network is defined before the first necessary change of nodes. Also, knowing that the change of nodes must occur or that this change is inevitable after some time, the optimal routing of mobile nodes has its importance. Each layout and routing of mobile sensor nodes strives to minimize the number of required sensor nodes while ensuring that efficiency is as high as possible. ${ }^{7}$

\section{CONCLUSION}

Extensive research on sensor networks boosts the evolution of these systems. Although sensornetworks are mostly considered as fixed topologies (with stationary sensor nodes), and sensor networkprotocols so far usually assume that the nodes are stationary, next generation sensor networks may beexpected to include mobile sensor nodes. As such systems evolve, mobile nodes will "carry" time information from one part of thenetwork to other parts, potentially increasing the global synchronization accuracy. Wireless sensor networks are an increasing support for autonomous systems. Given this, the quality of designing and building wireless sensor networks is very important. In order to maximize their functionality, optimal routing of mobile sensor nodes within these networks is crucial.

\section{AUTHORS}

First Author - Alaulddin Adel Albla Phd

alladdin@hiuc.edu.iq

Alhikma college University

\footnotetext{
5 Liu, J., Feng, Z., Petrovic, D. (2005) Information-directed routing in ad hoc sensor networks. IEEE Journal on Selected Areas in Communications, 23(4): 851-861

6 Bežične senzorske mreže u poljoprivredi (Wireless sensor networks in agriculture), A. Višnjički, Beograd,2020.

https://raf.edu.rs/docs/Diplomski_radovi/Aleksandar_Visnjicki_ Diplomski_rad.pdf,

${ }^{7}$ Optimal Routing Of Mobile Nodes In Wireless Sensor Networks, Mateo Leovac, 2019, https://core.ac.uk/download/pdf/270103071.pdf 
International Journal of Scientific and Research Publications, Volume 11, Issue 8, August 2021

This publication is licensed under Creative Commons Attribution CC BY. 